\title{
Radar and rocket comparison of UHF radar scattering from auroral electrojet irregularities: Implications for a nanosatellite radar
}

\author{
Hasan Bahcivan, ${ }^{1}$ Michael C. Kelley, ${ }^{2}$ and James W. Cutler ${ }^{3}$ \\ Received 4 February 2009; revised 31 March 2009; accepted 15 April 2009; published 17 June 2009. \\ [1] This article presents the estimates for UHF radar volume scattering coefficient of \\ auroral electrojet irregularities based on previous measurements of absolute radar \\ scattering coefficients and in situ rocket spectra. Both estimates indicate that a \\ low-earth-orbiting nanosatellite with a 0 -dB gain UHF antenna will be sensitive to \\ bistatic scatter into space from the irregularities illuminated by the ground-based Poker \\ Flat Incoherent Scatter Radar (PFISR). It is also shown that the change of scattering \\ geometry due to the satellite motion is sufficiently slow to "lock on" the target. That is, \\ the satellite will acquire a statistically large number of samples in a sufficiently small time \\ window to resolve the scattering signal power as a function of the Bragg scattering angle \\ with respect to the magnetic field lines.
}

Citation: Bahcivan, H., M. C. Kelley, and J. W. Cutler (2009), Radar and rocket comparison of UHF radar scattering from auroral electrojet irregularities: Implications for a nanosatellite radar, J. Geophys. Res., 114, A06309, doi:10.1029/2009JA014132.

\section{Introduction}

[2] The National Science Foundation has recently selected a radar-based system as their first nanosatellite. The satellite mission is Radio Aurora Explorer (RAX) and is a CubeSat-based ([Nason et al., 2002] 9-16 March) groundto-space bistatic radar experiment. It will be a collaborative effort of SRI International and the University of Michigan [Moretto, 2008]. The idea is to use a ground-based radar to illuminate the auroral electrojet and to receive the scattered signal with a simple dipole antenna on the satellite. The primary transmitting system is the $2 \mathrm{MW}, 450 \mathrm{MHz}$, phased-array Poker Flat Incoherent Scatter Radar (PFISR). Figure 1 is an illustration of the experiment.

[3] The ground-to-space bistatic geometry enables high horizontal and altitudinal resolution measurements of the auroral field-aligned irregularities (FAI), which has not been possible from the ground due to the difficulty associated with the magnetic field lines being nearly vertical. The high resolution is achieved through two factors: (1) the radar illuminating the irregularities has a narrow beam width, which is typical of all incoherent scatter radars (ISR), and (2) the radar-to-irregularity distance is short because the radar can be pointed at high elevation angles (as opposed to a monostatic radar pointed at very low elevation to meet the perpendicularity condition). Furthermore, PFISR will make incoherent scatter measurements of the electric field and the

\footnotetext{
${ }^{1}$ Center for Geospace Studies, SRI International, Menlo Park, California, USA.

${ }^{2}$ Department of Electrical and Computer Engineering, Cornell University, Ithaca, New York, USA.

${ }^{3}$ Department of Aerospace Engineering, University of Michigan, Ann Arbor, Michigan, USA.
}

Copyright 2009 by the American Geophysical Union. 0148-0227/09/2009JA014132 electron density in the same scattering volume, simultaneously characterizing the conditions that give rise to FAI.

[4] In this letter, we present radar sensitivity calculations for the RAX mission based on previous observations of absolute radar backscatter coefficients and in situ rocket spectra in the auroral $E$ region. First, we estimate RAX signal-to-noise ratios (SNR) based on previous radar-based measurements of absolute volume scattering coefficients. Second, we formulate the volume scattering coefficient from an irregularity $\mathrm{k}$ spectrum and estimate RAX SNR based on previous in situ rocket measurements of $\mathrm{k}$ spectra. Third, we compare the radar- and rocket-based estimates. We finally discuss the implications of the results for the RAX mission.

\section{Sensitivity Based on UHF Radar Backscatter Observations}

[5] The radar equation for the power at the satellite receiver input is

$$
P_{r}=\frac{P_{t} L G_{t}}{4 \pi R_{1}^{2}} \cdot V_{s} \sigma_{v} \cdot \frac{G_{r} \lambda^{2}}{4 \pi R_{2}^{2}(4 \pi)}=P_{t} L \cdot c \Delta \tau \sigma_{v} \cdot \frac{G_{r} \lambda^{2}}{4 \pi R_{2}^{2}(4 \pi)}
$$

where $P_{t}$ is the transmitted power, $L$ is the system loss, $G_{t}$ $\left(G_{r}\right)$ is the transmitting (receiving) antenna gain, $R_{1}\left(R_{2}\right)$ is the distance between the transmitter (receiver) and the irregularities, $V_{s}$ is the scattering volume, $\sigma_{v}$ is the effective volume scattering coefficient per unit volume (to be formally defined below), and $\lambda$ is the radar wavelength. Assuming beam-filled scatter for $\approx 1^{\circ}$ PFISR beam width, the radar equation simplifies as the RHS where $\Delta \tau$ is the radar pulse length. Figure 2 shows a schematic of the scattering geometry. 


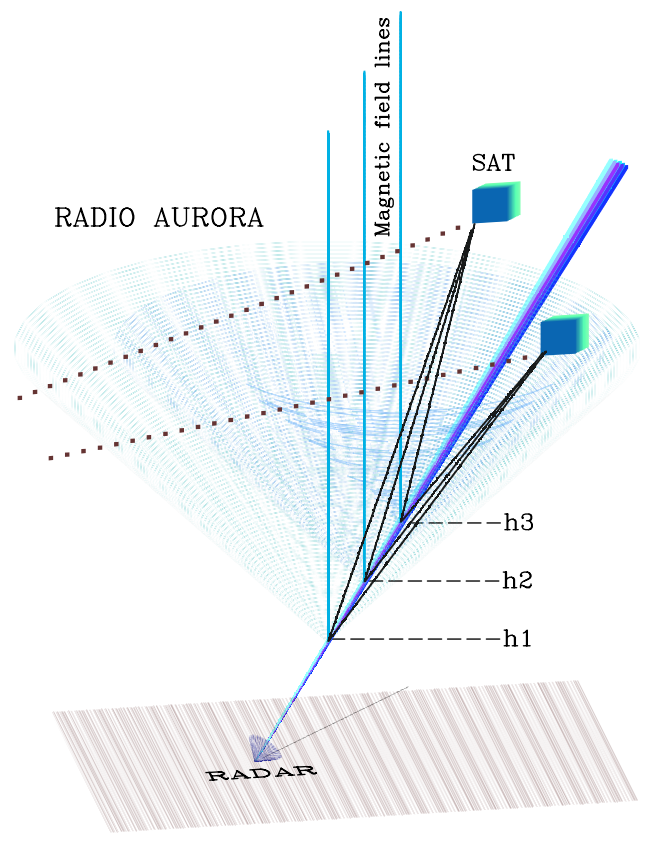

Figure 1. An illustration of the ground-to-space radar aurora experiment. The satellite tracks represent multiple passes.

[6] Table 1 shows the radar parameters for RAX and, for comparison, the Homer UHF radar system [Chestnut et al., 1968]. The received power is based on the expression

$$
\sigma_{v}^{\max }(k)=(4 \pi) \times 3.0 \times 10^{-7} k^{-2.25}\left(m^{-1}\right)
$$

which was derived by Moorcroft [1987] based on a set of $E$ region VHF-UHF radar absolute power measurements at a set of radar wave numbers $k$. Note that we multiplied the original expression for $\sigma_{v}^{\max }(k)$ by $4 \pi$ so that the definition of the radar scattering cross section is consistent with the radar equation (1). This empirical formula is for the upper boundary (highest SNR) of a statistical set of coefficients gathered for each $k$. Figure 3 shows the distribution of

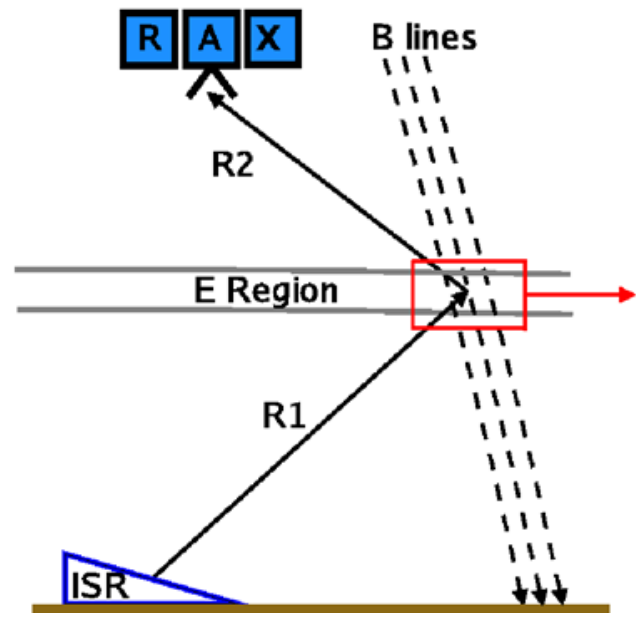

Figure 2. Ground-to-space bistatic scatter geometry representing the PFISR-RAX radar experiment. receiver power based on two separate time windows of Homer radar measurements. The bottom axis is shifted to interpret the data for RAX. The RAX noise power corresponds to a noise temperature of $400 \mathrm{~K}$ for a system looking toward the Earth [Baron et al., 1976]. For the PFISR-RAX geometry where the angle between the incident and scattered radar waves is $90^{\circ}$ (see Figure 2), the Bragg wave number is $\sqrt{2}$ times the radar wave number (Bragg wavelength $=0.47 \mathrm{~m}$ ), and the length of scattering along the incident radar beam is given by $c \Delta \tau$, as opposed to $c \Delta \tau / 2$.

[7] In calculating $\sigma_{v}$, Moorcroft [1987] assumed that the vertical thickness of the scattering layer is $10 \mathrm{~km}$, which for Homer's $800 \mathrm{~km}$ range means that scattering comes from between the aspect angles $\pm 0.36^{\circ}$. This range, which is comparable to the PFISR beam width, justifies the beamfilled scattering assumption we have just made for PFISR.

[8] A further note is that there may be polarizationdependent signal loss. Although the incident PFISR beam is circularly polarized, the eccentricity of the scattered waves depends on the scattering angle. A polarization eccentricity plot (not shown here) for the satellite zone of scattering shows the scattering to be between elliptical to linear (eccentricity between $0.8-1.0$ ). In the case of $90^{\circ}$ scattering angle, the scattered waves are linearly polarized, which means half of the scattered power is lost. Note that Table 1 does not include polarization-related losses, which will depend on the scattering geometry and the polarization of the receiving antenna.

\section{Sensitivity Based on In Situ Rocket Spectra 3.1. Volume Scattering Formalism}

[9] To compute the volume scattering coefficient $\eta$ from rocket in situ power spectrum measurements, we follow the technique pioneered by Woodman and Basu [1978], developed further by Hysell et al. [1994], and tested by Franz et al. [1999]. Defining $\left\langle|\delta n(\mathbf{k})|^{2}\right\rangle$ as the three-dimensional power spectrum, the volume scattering coefficient is obtained as

$$
\eta(\mathbf{k})=4 \pi r_{e}^{2}\left\langle|\delta n(\mathbf{k})|^{2}\right\rangle \quad\left(m^{-1}\right)
$$

where $r_{e}=2.8 \times 10^{-15} \mathrm{~m}$ is the classical electron radius.

Table 1. RAX Radar Sensitivity Compared With Homer Radar's for a Statistically Maximum Scattering Coefficient

\begin{tabular}{lccc}
\hline & RAX & Homer & Unit \\
\hline$P_{t}$ & $2 \times 10^{9}$ & $4 \times 10^{7}$ & $\mathrm{MW}$ \\
$L$ & 1.0 & 0.63 & - \\
$G_{t}$ & 20000 & 3981 & - \\
$\Delta \tau$ & 300 & 300 & $\mu \mathrm{s}$ \\
$V_{s}$ & $1.27 \times 10^{12}$ & $1.45 \times 10^{13}$ & $\mathrm{~m}^{3}$ \\
$\sigma_{v}$ & $1.11 \times 10^{-8}$ & $6.71 \times 10^{-9}$ & $\mathrm{~m}^{-1}$ \\
$G_{r}$ & 1 & 3981 & - \\
$\lambda$ & 0.670 & 0.754 & $\mathrm{M}$ \\
$R_{1}$ & 150 & 800 & $\mathrm{Km}$ \\
$R_{2}$ & 1000 & 800 & $\mathrm{Km}$ \\
$P_{r}$ & -82.5 & -75.7 & $\mathrm{DBm}$ \\
$P_{n}$ & -122.6 & -115.0 & $\mathrm{DBm}$ \\
$\mathrm{SNR}_{\max }$ & 40.1 & 39.3 & $\mathrm{DB}$ \\
\hline
\end{tabular}




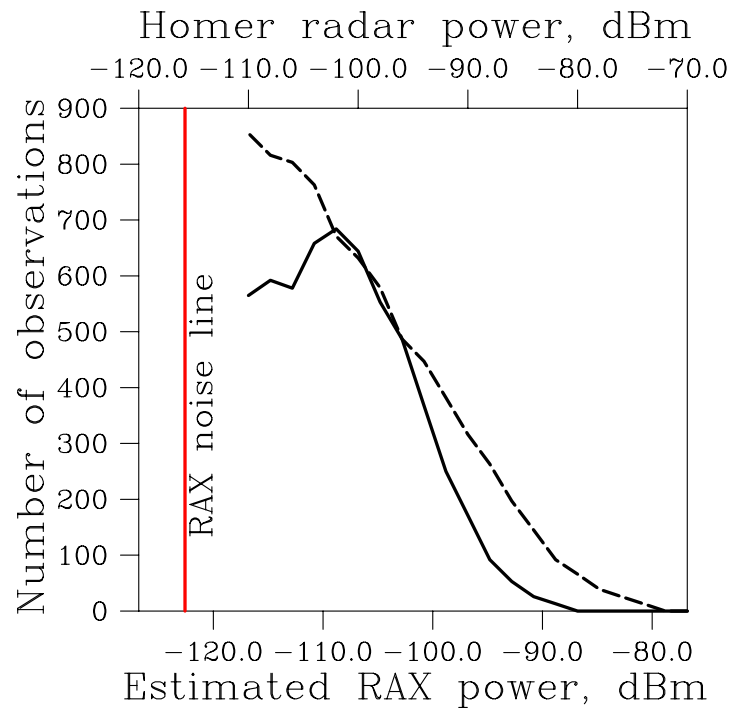

Figure 3. Distribution of received power based on two separate time windows of Homer radar measurements (from Moorcroft [1987]). The bottom axis is shifted to interpret the data for RAX.

[10] For field-aligned irregularities, Woodman and Basu [1978] modeled the 3D spectrum as a product of the 2D perpendicular spectrum and a parallel spectrum given by the delta function:

$$
\eta(\mathbf{k})=4 \pi r_{e}^{2}\left\langle\left|\delta n\left(\mathbf{k}_{\perp}\right)\right|^{2}\right\rangle \delta\left(k_{\|}\right)
$$

[11] Lets define a normalized two-dimensional perpendicular power spectrum $f\left(k_{\perp}\right)$ (with units $m^{2}$ ) such that

$$
\left\langle\left|\delta n\left(\mathbf{k}_{\perp}\right)\right|^{2}\right\rangle=\left\langle|\Delta N|^{2}\right\rangle f\left(k_{\perp}\right)\left(m^{-4}\right)
$$

and

$$
\int_{\theta} \int_{k_{\perp}} f\left(k_{\perp}\right) k_{\perp} d k_{\perp} d \theta=1
$$

Here $\left\langle\left|\delta n\left(\mathbf{k}_{\perp}\right)\right|^{2}\right\rangle$ is the mean square of broadband electron density fluctuations. Considering that Moorcroft's empirical power law form in (2) is that of an isotropic twodimensional spectrum (on the plane perpendicular to B), its normalized form (see equation (6)) is

$$
f\left(k_{\perp}\right)=0.087\left(k_{\perp} / k_{0}\right)^{-2.25}\left(m^{2}\right)
$$

for a scale $k_{0}=1 \mathrm{~m}^{-1}$. This scale is introduced to make the unit independent of the power law exponent. This function is plotted in Figure 4.

[12] Woodman and Basu [1978] integrate equation (4) in volume to obtain the total scattering cross section for perpendicular scattering as

$$
\sigma\left(k_{\perp}\right)=4 \pi r_{e}^{2}\left\langle|\Delta N|^{2}\right\rangle f\left(k_{\perp}\right) A_{\perp} \frac{R}{k} \quad\left(m^{2}\right)
$$

where $A_{\perp}$ is the area of the zero-aspect angle intersection of the radar scattering volume with the $B_{\perp}$ plane, and $R$ is the range to the target. Note that there is neither $k_{\|}$ dependence in the above formula nor a dependence on the aspect sensitivity of the irregularities. This result comes from the choice of aspect dependence given by $\delta\left(k_{\|}\right)$. Then, equation (8) approximately holds if the radar beam width is larger than or comparable to the irregularity aspect width $\left(\approx 1^{\circ}\right)$, and we assume this is the case here, at least for electric fields $<40 \mathrm{mV} / \mathrm{m}$. In the presence of stronger electric fields, the irregularity spectrum is expected to widen in aspect angle, being broader than the radar beam width. The treatment of such cases will require beam patternweighted integration over aspect angle.

[13] Finally, as the basis for the rocket-radar backscatter comparison in the next sections, we define the effective volume scattering coefficient as

$$
\sigma_{v}=\sigma\left(k_{\perp}\right) / V_{s} \quad\left(m^{-1}\right)
$$

where $V_{s}=A_{\perp} d$ is the radar scattering volume, the volume of the plasma inside a radar's range gate, and $d$ is the thickness of the scattering region. This definition is necessary because $\eta(\mathbf{k})$ is strongly aspect sensitive with respect to the magnetic field and the backscatter to the radar will be a weighted average of $\eta$ over a range of aspect angles. Therefore, combining (8) and (9),

$$
\sigma_{v}=4 \pi r_{e}^{2}\left\langle|\Delta N|^{2}\right\rangle f\left(k_{\perp}\right) \frac{R}{k d}
$$

[14] At this point, the reader may be wondering why we use $V_{s}=A_{\perp} d$ in place of the scattering volume for RAX. We do so, so that we can compare $\sigma_{v}$ derived from Homer radar measurements to $\sigma_{v}$ derived from rocket measurements for

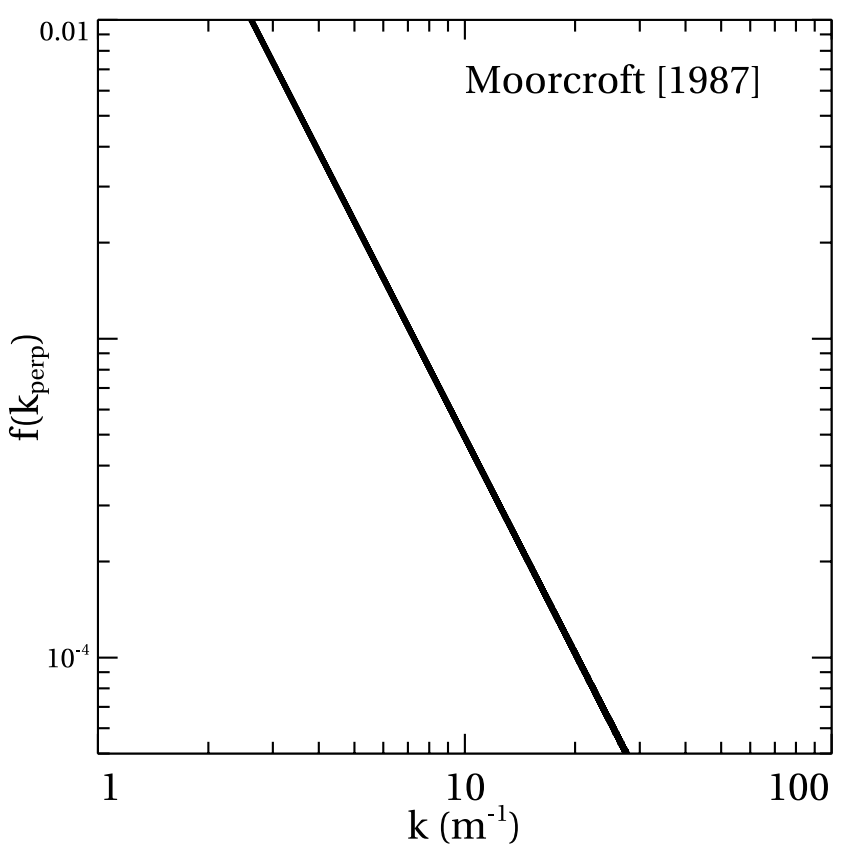

Figure 4. Normalized two-dimensional power spectrum $f\left(k_{\perp}\right)$ (equation (7)) corresponding to the radar-radar $\mathrm{k}$ spectrum (equation (2)). 

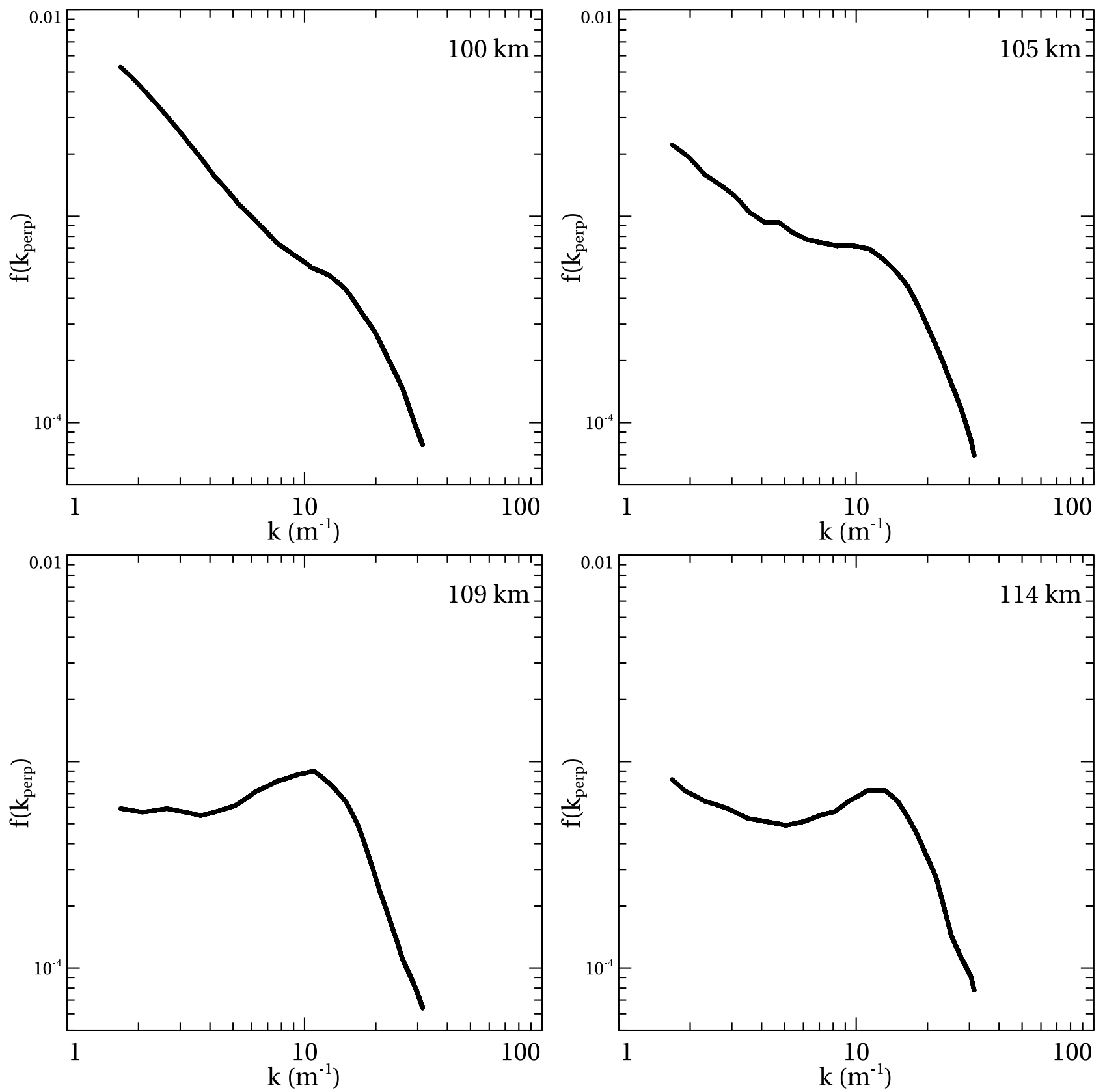

Figure 5. Auroral $E$ region electric field power spectra from ROSE F4 rocket data (after normalization).

the same assumption of scattering thickness. We will then use rocket-based $\sigma_{v}$ to calculate the received power by RAX.

\subsection{Electric Field and Electron Density Power Spectra}

[15] Computing equation (9) from the rocket power spectrum requires that we determine the density spectrum $\left\langle\left|\delta n\left(\mathbf{k}_{\perp}\right)\right|^{2}\right\rangle$ first. According to the linear theory (equation 4.39 of Kelley [1989]), the electron density fluctuation $\delta n$ is related to the electric field fluctuation $\delta E_{x}$ as

$$
\begin{aligned}
\delta E_{x} / E_{z 0} & =\chi \delta n / n \\
\chi(k) & =\left[\frac{\Psi_{0}\left(\Omega_{e}^{2}+\nu_{e}^{2}\right)}{\nu_{e} \Omega_{e}\left(1+\Psi_{0}\right)}+i \frac{k k_{B} T_{e}}{e E_{z 0}}\right]
\end{aligned}
$$

where $v_{e, i}$ are the electron and ion collision frequencies; $\Omega_{e, i}$ are the electron and ion gyrofrequencies; $E_{z 0}$ is the convection electric field; $\Psi_{0}=v_{e} v_{i} / \Omega_{e} \Omega_{i}$.

[16] Using (11), the density spectrum can be obtained from the electric field spectrum as

$$
\left\langle|\delta n(k)|^{2}=\right\rangle\left\langle|\delta E(k)|^{2}\right\rangle /|\chi(k)|^{2}
$$

In the following, we first obtain $\left\langle|\delta E(k)|^{2}\right\rangle$ from rocket measurements and use $E_{z 0}=40 \mathrm{mV} / \mathrm{m}, v_{e}=10000 \mathrm{~s}^{-1}, v_{i}=$ $2500 \mathrm{~s}^{-1}$, and $T_{e}=800 \mathrm{~K}$ to get $\left\langle|\delta n(k)|^{2}\right\rangle$. The collision frequencies are representative of the altitude of $105 \mathrm{~km} ; E_{z 0}$ value is what was measured during the ROSE F4 rocket (see below); $T_{e}$ value reflects the approximately linear increase 

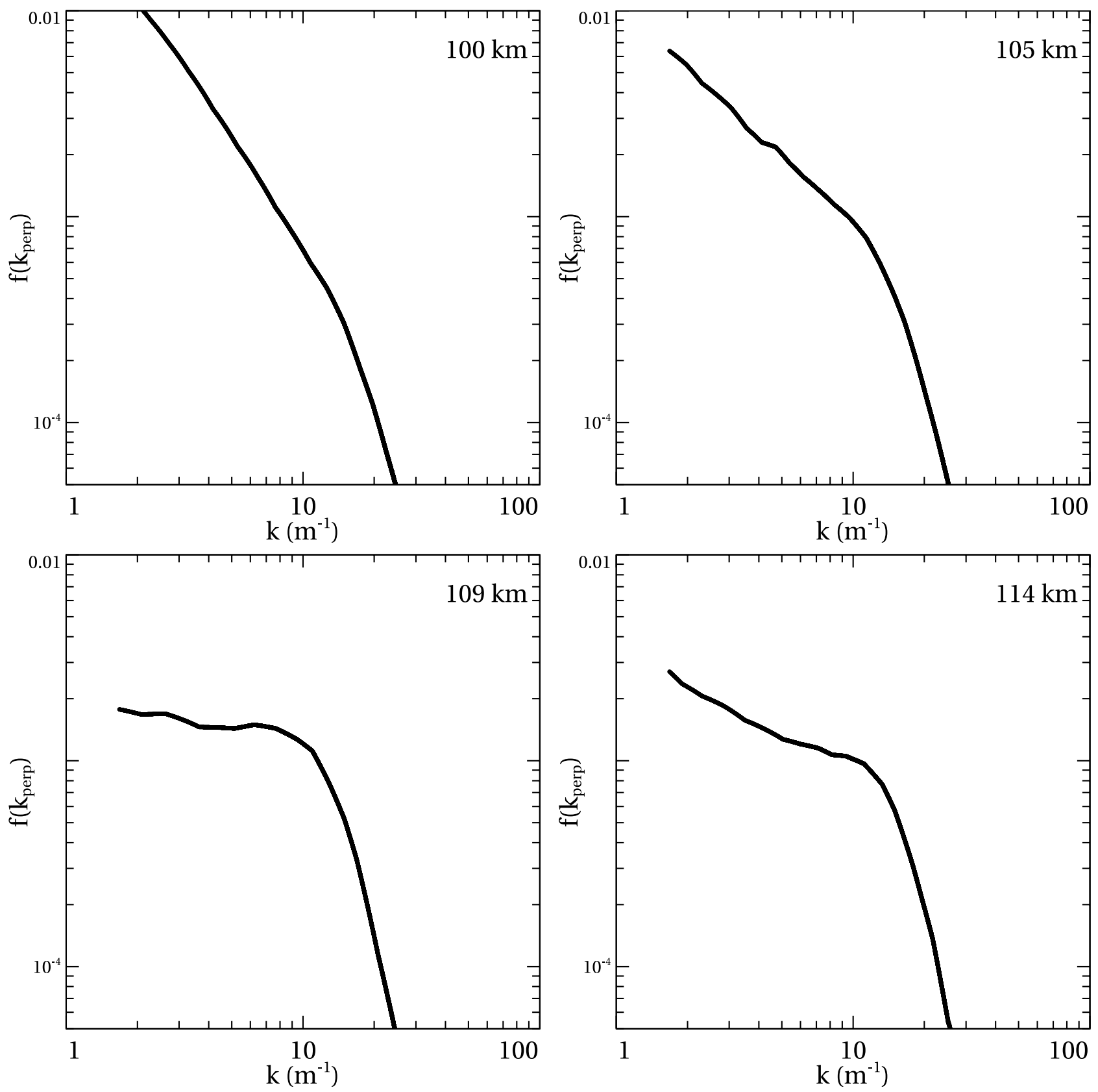

Figure 6. Auroral $E$ region electron density power spectra from ROSE F4 rocket electric field data and equation (12) (after normalization).

of the electron temperature with the electric field, i.e., $20 \mathrm{~K}$ per $\mathrm{mV} / \mathrm{m}$ [Bahcivan et al., 2006].

[17] To evaluate the electric field power spectrum at the $47-\mathrm{cm}$ scale is tricky if the electric field antenna is more than twice this scale. This is the case for the auroral rocket measurements with several-meter antennas [e.g., Pfaff et al., 1985]. We are first going to use the auroral rocket spectral data representing decimeter and larger scales from the Rocket and Scatter Experiments (ROSE) campaign [Rose, 1992] and then use a simple spectral model representing previous rocket observations with meter and longer antennas.

[18] The KUSO electric field probe on the ROSE F4 rocket was a $0.23 \mathrm{~m}$ diameter sphere with the surface segmented into electrically isolated regions. The electric field measurements were made by measuring the voltage difference between opposing surfaces. Because the effective antenna size $(<0.23 \mathrm{~m})$ is shorter than the Bragg scale $(0.47 \mathrm{~m})$, the antenna filtering is not significant.

[19] Figure 5 shows the normalized two-dimensional electric field power spectrum $f\left(k_{\perp}\right)$ for the ROSE F4 rocket. The plots are for four different altitudes in the auroral $E$ region. At the time of the $\mathrm{F} 4$ flight, the $E \times B$ drift was $\approx 800 \mathrm{~m} / \mathrm{s}$. Considering that the waves propagate at the ionacoustic velocity $(\approx 500 \mathrm{~m} / \mathrm{s})$ in the direction of the $E \times B$ drift and considering the rocket velocity reported in the study by Rose [1992], we estimated that the irregularities move at $\approx 600 \mathrm{~m} / \mathrm{s}$ in the rocket frame. Then, the frequency axis is converted to the $k$ axis as $k=2 \pi f / 600 \mathrm{~m}^{-1}$. Lastly, 


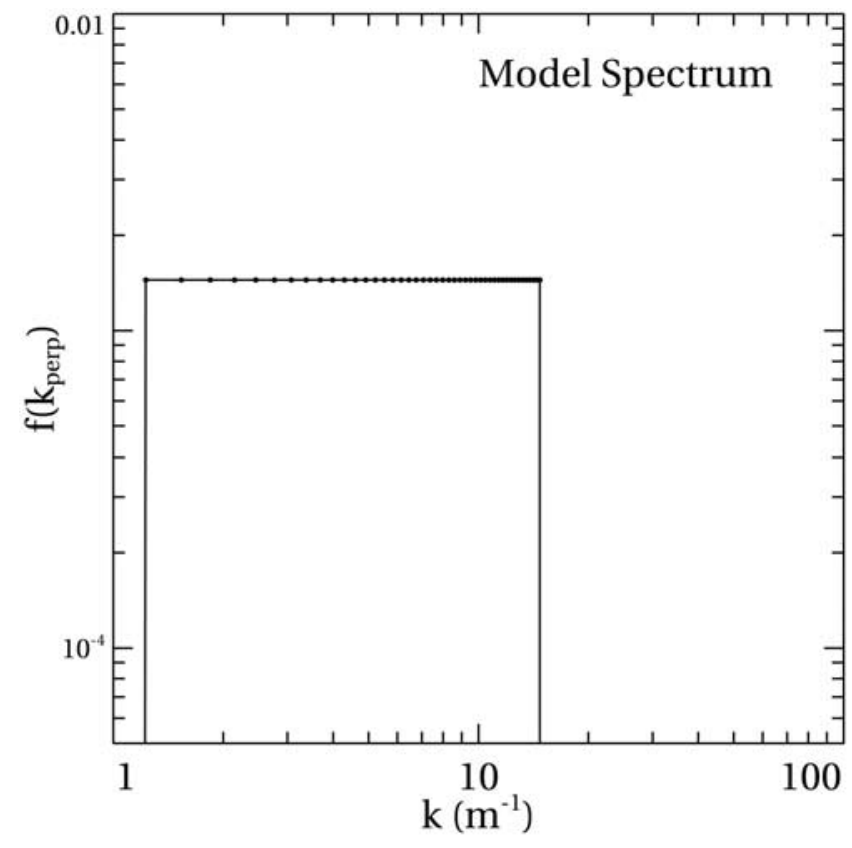

Figure 7. Model electron density power spectrum.

the spectra were normalized so that the two-dimensional integral over $k_{\perp}$ is equal to unity (see equation (6)).

[20] Figure 6 shows the normalized two-dimensional density spectrum constructed using the data in Figure 5 and the equation (12).

[21] We now develop a simple model of $\mathrm{k}$ spectrum representing the measurements made by meter-scale rocket antennas. Figure 5 of Pfaff et al. [1985] shows that most of the energy of the rocket electric field spectrum in the auroral $E$ region is located at the meter scales and the spectrum evolves into white for smaller scales. However, since the spectra at the sub-meter scales are suppressed, we cannot evaluate these spectra for the $47 \mathrm{~cm}$ wavelength. Instead, we will represent the meter-scale antenna measurements with a simple spectrum that is flat between $k=1 \mathrm{~m}^{-1}$ and $k=$ $15 \mathrm{~m}^{-1}$ and zero elsewhere, as shown in Figure 7.

\section{Comparison of Backscatter Strength}

[22] To compare the radar and rocket-based estimates of $\sigma_{v}$, we use the Homer radar geometry, which is monostatic with $R=800 \mathrm{~km}$, and a scattering layer thickness $\left(\mathrm{V}_{s} / \mathrm{A}_{\perp}\right)$ of $10 \mathrm{~km}$ (these are what Moorcroft [1987] used for the Homer radar). However, the Bragg wave number will be that of the PFISR-RAX bistatic radar $(0.47 \mathrm{~m})$.

[23] Assuming 3\% density fluctuations, $3 \times 10^{11} \mathrm{~m}^{-3}$ mean electron density, and an isotropic $\mathrm{k}$ spectrum in the plane perpendicular to $B$, we obtain $\sigma_{v}$ (from equation (9)) as shown in Table 2. Note that $\sigma_{v}$ from the normalized radar spectrum is obtained by using the normalized $k^{-2.25}$ form, which is equation (7), and the assumed density fluctuation $(3 \%)$ in the expression (10). The assumed $3 \times 10^{11} \mathrm{~m}^{-3}$ mean electron density is in the range of electron density, $\approx 1-4 \times 10^{11} \mathrm{~m}^{-3}$, measured by the European Incoherent Scatter Radar (EISCAT) between the altitudes of 100$110 \mathrm{~km}$ during the four rocket flights of the ROSE cam- paign [Kohl et al., 1992]. In addition, the assumed 3\% electron density fluctuation is in the range of $2-4 \%$, as measured by the ROSE rockets for the same altitude range [Schlegel, 1992].

[24] Table 2 shows that the effective volume scattering coefficients from a simple flat spectral model and the ROSE rocket $\mathrm{k}$ spectrum data from the KUSO probe and from the normalized radar spectrum match within $5.7 \mathrm{~dB}$. The $\sigma_{v}^{\max }$ from the original radar spectrum is $24.8 \mathrm{~dB}$ higher than the highest rocket-based $\sigma_{v}$. As the right two columns of Table 2 show, the corresponding RAX $P_{r}$ and SNR will differ by the same factors. In all cases, the rocket- and model-based $P_{r}$ is within the statistical range of the radarbased power distribution shown in Figure 3. Note that the peak of the distribution (displayed by the solid line) is located at $\sim-110 \mathrm{dBm}$.

[25] It is known that the $\mathrm{k}$ spectrum is not isotropic in the plane perpendicular to the magnetic field, but it is stronger in the $E \times B$ direction. This has been observed during the JOULE I joint rocket and radar campaign [Bahcivan et al., 2005], by both set of auroral rockets [Pfaff et al., 1985; Rose, 1992], and at the magnetic equator [Pfaff et al., 1987]. The Moorcroft formula is assumed to be for primary irregularities (in the $E \times B$ direction). Our calculation from in situ rocket spectra is for an isotropic $\mathrm{k}_{\perp}$ spectrum, and therefore will underestimate the scattering intensity.

[26] A final note is that radar soundings of plasma irregularities are random, and therefore, a large number of echoes must be received to statistically determine the scattering amplitudes. We have determined that the scattering aspect angle change due to the satellite motion is $\sim 0.15^{\circ}-0.30^{\circ} \%$ s near the loci of perpendicular scattering. This means, for an interpulse period (IPP) of $10 \mathrm{~ms}, 100$ pulses can be transmitted for each $0.3^{\circ}$ wide aspect angle bin. Considering that the maximum Doppler width of the $E$ region irregularities were observed to be a few $100 \mathrm{~m} / \mathrm{s}$ [Bahcivan et al., 2005], the correlation time of the irregularities are less than $1 \mathrm{~ms}$ at the Bragg wavelength of $0.47 \mathrm{~m}$. Therefore the 100 pulses are uncorrelated and the statistical uncertainty of the wave amplitudes will be $10 \%$. Furthermore, the aspect angle distribution within the PFISR beam width for an $E$ region range gate $\left(3 \mathrm{~km}\right.$ long) is about $0.5^{\circ}$ wide. Considering that the aspect angle widths of auroral echoes will be $1^{\circ}$ or larger and excluding radar sidelobe effects, the PFISR-RAX system with a $10 \mathrm{~ms}$ IPP should resolve the auroral $E$ region irregularities with $0.5^{\circ}$ angular resolution and $10 \%$ uncertainty. We do realize that the large dynamic range of auroral echoes and their strong aspect

Table 2. Effective Volume Scattering Coefficients and the Corresponding RAX Received Power and SNR

\begin{tabular}{lccc}
\hline & $\sigma_{v}-\mathrm{m}^{-1}$ & $\mathrm{P}_{r}-\mathrm{dBm}$ & SNR-dB \\
\hline Rocket & & & \\
$100 \mathrm{~km}$ & $1.9 \times 10^{-11}$ & -110.2 & 12.4 \\
$105 \mathrm{~km}$ & $2.7 \times 10^{-11}$ & -108.6 & 14.0 \\
$109 \mathrm{~km}$ & $3.4 \times 10^{-11}$ & -107.6 & 15.0 \\
$\quad 114 \mathrm{~km}$ & $3.7 \times 10^{-11}$ & -107.3 & 15.3 \\
Flat spectra & $7.0 \times 10^{-11}$ & -104.5 & 18.1 \\
Radar spectra, normal & $1.2 \times 10^{-11}$ & -112.2 & 10.4 \\
Radar spectra, original & $8.8 \times 10^{-10}$ & -82.5 & $40.1^{\text {max }}$ \\
\hline
\end{tabular}


sensitivity will present a challenge to achieving the $0.5^{\circ}$ angular resolution. Nevertheless, we anticipate that this resolution can be achieved by an advanced analysis involving the deconvolution of the PFISR beam pattern by combining the satellite measurements made at consecutive points along the orbit. A manuscript on this technique is in preparation.

[27] In conclusion, both the radar- and rocket-based SNR show that the RAX satellite will be sensitive to the coherently scattered PFISR radar signals from field-aligned auroral electrojet irregularities. Because PFISR can measure the plasma density and the electric field $E$ in the scattering volume effectively simultaneously, the amplitude of the waves at the radar Bragg scale as a function of $E$ can be determined. Furthermore, simultaneous measurements of plasma temperatures by PFISR can elucidate the plasma wave heating [Bahcivan, 2007] as a function of $E$ and wave amplitudes.

[28] Acknowledgments. This work was supported by NSF grant ATM-0838054 to SRI International and the University of Michigan.

[29] Amitava Bhattacharjee thanks Donald R. Moorcroft and another reviewer for their assistance in evaluating this paper.

\section{References}

Bahcivan, H. (2007), Plasma wave heating during extreme electric fields in the high-latitude E region, Geophys. Res. Lett., 34, L15106, doi:10.1029/ 2006 GL029236.

Bahcivan, H., D. L. Hysell, M. F. Larsen, and R. F. Pfaff (2005), The $30 \mathrm{MHz}$ imaging radar observations of auroral irregularities during the JOULE campaign, J. Geophys. Res., 110, A05307, doi:10.1029/ 2004JA010975.

Bahcivan, H., R. Cosgrove, and R. Tsunoda (2006), Parallel electron streaming in the high-latitude E region and its effect on the incoherent scatter spectrum, J. Geophys. Res., 111(A7), A07306, doi:10.1029/ 2005JA011595.

Baron, M., R. Tsunoda, J. Petriceks, and H. Kunnes (1976), Feasibility of an incoherent-scatter radar aboard the space shuttle, Stanford Res. Inst. Final Rep., SRI Proj. 4278.
Chestnut, W. G., J. C. Hodges, and R. L. Leadabrand (1968), Auroral backscatter wavelength dependence studies, SRI Proj. 5535 Final Rep., Stanford Res. Inst., Menlo Park, Calif.

Franz, T. L., M. C. Kelley, and A. V. Gurevich (1999), Radar backscattering from artificial field-aligned irregularities, Radio Sci., 34(2), 465-475.

Hysell, D. L., M. C. Kelley, W. E. Swartz, and D. T. Farley (1994), VHF radar and rocket observations of equatorial spread $F$ on Kwajalein, J. Geophys. Res., 99(A8), 15,065-15,085.

Kelley, M. C. (1989), Earth's Ionosphere, Plasma Physics and Electrodynamics, Acad. Press, New York.

Kohl, H., E. Nielsen, K. Rinnert, and K. Schlegel (1992), EISCAT results during the ROSE campaign and comparison with STARE measurements, J. Atmos. Terr. Phys., 54, 733-739.

Moorcroft, D. R. (1987), Estimates of absolute scattering coefficients of radar aurora, J. Geophys. Res., 92(A8), 8723-8732.

Moretto, T. (2008), CubeSat mission to investigate ionospheric irregularities, Space Weather, 6, S11002, doi:10.1029/2008SW000441.

Nason, I., J. Puig-Suari, and R. Twiggs (2002), Development of a family of picosatellite deployers based on the CubeSat standard, in Proc. of the 2002 IEEE Aerospace Conf., 1, Big Sky, Mont., 9-16 March.

Pfaff, R. F., M. C. Kelley, B. G. Fejer, N. C. Maynard, L. G. Brace, B. G. Ledley, L. G. Smith, and R. F. Woodman (1985), Comparative in situ studies of the unstable daytime equatorial $E$ region, J. Atmos. Terr. Phys., $47,791-811$.

Pfaff, R. F., M. C. Kelley, E. Kudeki, B. G. Fejer, and K. D. Baker (1987), Electric field and plasma density measurements in the strongly driven daytime equatorial electrojet: 2. Two-stream waves, J. Geophys. Res., 92(A12), 13,597-13,612.

Rose, G. (1992), Three-component a.c. E-field observations during the rocket and scatter experiment in 1988-1989 under radar auroral conditions in Northern Scandinavia, J. Atmos. Terr. Phys., 54(6), 669-682.

Schlegel, K. (1992), Measurements of electron density fluctuations during the ROSE rocket flights, J. Atmos. Terr. Phys., 54, 715-723.

Woodman, R. F., and S. Basu (1978), Comparison between in-situ spectral measurements of $F$ region irregularities and backscatter observations at 3m wavelength, Geophys. Res. Lett., 5(10), 869-872.

H. Bahcivan, Center for Geospace Studies, SRI International, 333 Ravenswood Avenue, G278, Menlo Park, CA 94025, USA. (hasan.bahcivan@ sri.com)

J. W. Cutler, Department of Aerospace Engineering, University of Michigan, 1320 Beal Avenue, 3013 Francois Xavier Bagnoud Building, Ann Arbor, MI 48109-2140, USA.

M. C. Kelley, Department of Electrical and Computer Engineering, Cornell University, 318 Rhodes Hall, Ithaca, NY 14853, USA. 